\title{
ARTICULACION TERRITORIAL Y ESPACIOS NATURALES: UN ENSAYO PARA EL MODELO SOCIOECONOMICO DEL BAJO GUADALQUIVIR
}

Fernando DIAZ del OLMO *

Joaquin GUZMAN CUEVAS **

\section{INTRODUCCION Y SIGNIFICACION DEL TERRITORIO}

No cabe duda de que uno de los pilares sobre los que descansa la organización espacial es el transporte. La circulación terrestre, marítima o aérea, configura uno de los elementos más dinámicos de todo sistema territorial incidiendo considerablemente en la localización de las actividades económicas, desde agrícolas a las de ocio, perfilando la coligación del espacio, jerarquizando la red de rutas y en definitiva articulando el territorio. Precisamente la región andaluza no se distingue por su funcionalidad ni por su articulación $^{1}$, y lo mismo cabe apuntar para la tríada Huelva-Sevilla-Cádiz ${ }^{2}$, tanto como región económica cuanto en lo referente a infraestructuras viarias, particularmente carreteras locales y secundarias. Será en ésta segunda vertiente por la que desarrollaremos el tema del presente artículo, aunando, como ya hiciéramos en otras ocasiones ${ }^{3}$ los esfuerzos de las disciplinas que se vinculan en esta revista: Geografía y Economía.

La problemática de los transportes y circulación en Andalucía occidental, en tanto que inter-relaciones, no cuenta con lo que pudiéramos llamar un documento o estudio marco, muy necesario a todas luces. La Junta de Andalucía a través de la Consejería correspondiente, organizó en marzo de 1982 un Seminario-Coloquio sobre Infraestructura y Transporte de la región,

* Profesor de Geografía. Universidad de Sevilla.

** Profesor de Estructura y Política Económicas. Universidad de Sevilla.

1. CANO GARCIA, G. (1983) "Unidad y diversidad de la Geografía andaluza" Revista de Estudios Andaluces, n. ${ }^{\circ} 1$, pp. 9-22.

2. GONZALEZ DORADO, A. (1975): Sevilla: Centralidad regional y organización interna de un espacio urbano, Ser. Estudios del Banco Urquijo, Sevilla.

3. GUZMAN CUEVAS, J. Y DIAZ DEL OLMO, F. (1983): "Aportación al estudio de la organización del espacio andaluz: aplicación de un modelo gravitacional", Actas del VI Coloquio de Geografí, Palma de Mallorca (1979), pp. 559-565. 
y en diciembre-enero del 83-84 unas jornadas sobre el Transporte en el Are: de Sevilla. Otras aportaciones generales, locales o parciales al tema se abor dan en los volúmenes que dirigieran en 1978 y 1982 los profesores Bosque J Velarde, respectivamente, sobre la configuración e interpretación socioeco nómica de Andalucía. A tales estudios hay que añadir otros de índol proyectiva como los de Bernal, Titos, Guzmán y Díaz del Olmo ${ }^{4}$, o descrip tiva como el de Campos Romeros .

En el Bajo Guadalquivir se inscriben diversos complejos físicos, naturales demográficos y socioeconómicos. Desde las macrounidades geomorfológica de zócalo - Sierra Morena con o sin cobertera detrítica-, a los dominio sedimentarios mioplio-pleistocénicos - las comarcas del Condado, Campo di Tejada, Aljarafe-, los bajos tramos fluviales del Guadalquivir, Tinto, Odiel : Piedras, a los ámbitos litorales; pasando por los geosistemas de transició marismeños o estuarinos, dunares, o más o menos circunmediterráneos; todı el mosaico geográfico se manifiesta regularmente compartimentado. En ést. aproximación territorial hay que destacar la presencia de espacios naturales con planeamiento, o sin él en la actualidad, de protección integral o especia] Desde la desembocadura del Guadalquivir y sectores aledaños, hasta la de Guadiana por la costa, y limitando al Norte con las estribaciones mariánicas Huelva, y subsidiariamente Sevilla y Cádiz, cuentan con: el entorno d Doñana más las marismas de Bonanza, La Rocina, El Abalario y El Asperillc la ria y marisma del Odiel; la del Piedras con la flecha litoral de El Rompidc la laguna de El Portil; y las marismas de Isla Cristina.

En lo que atañe a tales espacios hemos de separar al presente el Parqu Nacional de Doñana - Parque y Reserva- con la Rocina -incluida en $\epsilon$ Pre-parque-, de los otros.

El Parque fue creado en agosto de 1969 viendo ampliar su planimetrí con delimitación de un área pre-parque en 1978, totalizando las $73.000 \mathrm{Has}$ actuales $^{7}$. Los complejos restantes se proponen en el catálogo de proteccic nes del Plan Especial de Protección del Medio Físico de Huelva -y Cádizactualmente en redacción.

4. BERNAL, A.M. (1973): "Area de influencia urbana de Sevilla según la movilidad de viajeros Estudios Geográficos, n. ${ }^{\circ} 131$, pp. 359-380. TITOS MORENO, A. (1975) "Consideraciones ace: ca del binomio planificación-infraestructura viaria en Andalucía" Información Comercial Españ la, n. ${ }^{0}$ 503, pp. 54-59. GUZMAN Y DIAZ DEL OLMO, Ob.cit.

5. CAMPOS ROMERO, M.L. (1983): "El transporte por carretera en la provincia de Córdoba Actas del VI Coloquio de Geografía, Palma de Mallorca (1979), pp. 515-522.

6. Interpretamos espacio natural en el mismo sentido que lo hace ANGLADA GOTOR, S. en su tr: bajo Aportación de la Ley de Doñana a los Parques Nacionales españoles, Temas Monográficc sobre Medio Ambiente (1979), n. ${ }^{\circ}$ 2, Junta de Andalucía, Granada.

7. Bibliografía sobre la historia y caracterización de Doñana hay mucha. De ella hemos escogidc RUBIO RECIO, J.M. (1977) "Nota sobre la significación biogeográfica y los problemas de la marismas del Guadaiquivir" Cuad. Geogr. de la Univ. Granada, n. ${ }^{0} 7$, pp. 277-293. AGUILA AMAT, J. y otros (1979) Parque Nacional de Doñana. Mapa Ecológico, ICONA. 
Desde un punto de vista demográfico se ha subrayado la vitalidad regional del triángulo Huelva-Sevilla-Cádiz muy recientemente ${ }^{8}$. En él resalta el crecimiento de los municipios costeros, del Condado y del área peri-urbana de Huelva ${ }^{9}$, directamente relacionados con las actividades turísticas, pesquerías, Plan Almonte-Marismas, vitivinicultura o el Polo Industrial onubense. Es de todas las comarcas la denominada Condado-Litoral -formada por Ios municipios de Almonte, Hinojos, Lucena del Puerto, Moguer y Palos de la Frontera-, la que galvaniza con mayor agudeza la dualidad que enfrenta articulación con desorganización territorial ${ }^{10}$.

\section{UN PERFIL SOCIOECONOMICO DEL BAJO GUADALQUIVIR}

Lógicamente al intentar desarrollar alguna valoración sobre el grado de integración de las comunicaciones, en cualquiera de sus facetas, de una determinada región geográfica, es insoslayable hacer unas referencias por breves que sean, al medio socioeconómico que condiciona o incluso determina la actividad intracomunicativa del área en cuestión. Area que por exigencias de las magnitudes estadísticas y sobre todo por la estrecha influencia que recibe de la unidad geoeconómica superior, nos obliga a ampliar nuestra óptica al ámbito territorial que conforman las tres provincias en las que se inserta el Bajo Guadalquivir.

La anterior alusión al fenómeno causa-efecto también se produce en nuestro contexto en sentido contrario: las limitaciones a una conveniente articulación de las vias de comunicaciones juegan en muchos casos $-\mathrm{y}$ el espacio andaluz como ya hemos apuntado es un claro exponente de ello-, un papel muy relevante en los mecanismos que frenan y distorsionan la dinámica del desarrollo.

8. JORDA BORRELL, R. (1983): "Areas de crecimiento demográfico en Andalucía" Revisto de Estudios Andaluces, n. ${ }^{\circ} 1$, pp. 43-58.

9. MONTEAGUDO LOPLZ-MENCHERO, J. (1980): Evolución geodemográfica de un sector periurbano de Huelva, Palos de la Frontera y Moguer 1960-1975. Publ. Colegio Universitario de La Rábida (Huelva).

10. Parte de esta problemática queda recogida en OJEDA RIVERA, J. y DIAZ DEL OLMO, F. (1983): "Configuración, determinismo natural y prelitoral onubense. El papel de la agricultura" Actas II Coloquio de Geografía Agraria, Memoria de Excursión, La Rábida, 1983. (En prensa). A este respecto es interesante el hecho de que en nuestro país los primeros proyectos de PDTC -Planes Directores Territoriales de Coordinación-, se han formulado para la comarca de Doñana y Gredos, ya que el PDTC se presenta según la legislación urbanística española como el instrumento de planificación y organización del territorio, con una clara incidencia en la protección medioambiental (vid. T.U.P.A., Urbanismo, Planeamiento y Arquitectura (1981) Proyecto de Plan Director Territorial de Coordinación de la Comarca de Doñana, M.O.P.U.-C.E.O.T.M.A.). 
El marco geográfico considerado muestra a nuestro juicio un perfil socioeconómico que refleja una clara correspondencia entre las variables desarrollo-comunicaciones. Conocido es que dentro del escalafón nacional, y para cualquier indicador de desarrollo que elijamos, las provincias que enmarcan al Bajo Guadalquivir se sitúan en los últimos lugares, en lógica consonancia con el exigüo nivel de la economía andaluza. No obstante dentro del contexto regional presentan algunas particularidades que vienen a poner de manifiesto en mayor o menor medida, la fuerte relación existente entre la desarticulación de las comunicaciones y el subdesarrollo económico.

Efectivamente, es suficientemente conocido que el triángulo SevillaCádiz-Huelva no alcanza las medias nacionales en los índices representativos de los niveles económicos: ingreso per cápita, renta familiar disponible, V.A.B. de su producción, desempleo, etc. Es más desde una perspectiva dinámica es también sabido que la posición relativa de cada una de las tres provincias en la escala nacional ha retrocedido sensiblemente en los últimos veinticinco años. A este tenor cabe subrayar que los índices de crecimiento de magnitudes tan significativas cara a las expectativas de futuro, tales como los ingresos totales o ingresos percapita muesatran, excepto para el caso de Sevilla, unos índices muy por debajo de las medias nacionales.

\section{CUADRO I}

PORCENTAJES DE INCREMENTO (1977-79)

\begin{tabular}{lcc}
\hline Provincias & Ingresos totales & Ingresos per cap. \\
\hline & & \\
Almería & 43,9 & 40,6 \\
Cádiz & 42,6 & 39,3 \\
Córdoba & 52,3 & 64,5 \\
Granada & 53,7 & 52,1 \\
Huelva & 36,3 & 34,2 \\
Jaén & 58,1 & 59,7 \\
Málaga & 56,3 & 49,5 \\
Sevilla & 49,6 & 45,6 \\
ESPA ÃNA & 47,4 & 44,3 \\
\hline
\end{tabular}

Fuente: Renta Nacional 1979. Banco de Bilbao.

Sin embargo estos bajos valores de progreso económico no guardan una línea de correspondencia coherente u ortodoxa con el modelo de crecimiento -aunque no necesariamente de desarrollo-, que rige en la mayor parte de las economías occidentales en general y en la española en particular. Es decir, un crecimiento caracterizado por el liderazgo del sector industrial que, principalmente y como consecuencia de su mayor aportación al V.A.B. de la 
producción total, representa en gran medida la fuerza motriz que arrastra a los otros dos macrosectores productivos y al conjunto de la economía en general.

En virtud de la distribución sectorial del V.A.N. (Valor Añadido Neto) para las distintas provincias andaluzas (cuadro II), se puede observar que a excepción hecha de Jaén, las tres del Bajo Guadalquivir poseen los mayores componentes industriales del V.A.N. siendo las únicas que superan los valores globales andaluces. Si nos atenemos al peso específico del V.A.N. industrial de cada una de las provincias andaluzas en relación al conjunto nacional, también observamos que, junto a Málaga, Sevilla, Cádiz y Huelva obtienen los valores absolutos más relevantes.

CUADRO II

DISTRIBUCION SECTORIAL DEL V.A.N. EN ANDALUCIA, 1979

\begin{tabular}{lcrrrrr}
\hline \multirow{2}{*}{ Provincias } & \multicolumn{2}{c}{ Agricultura y Pesca } & \multicolumn{2}{c}{ Industria } & \multicolumn{2}{c}{ Comercio y Servicios } \\
\cline { 2 - 7 } & Dist. Prov. & \% Nac. & Dist. Prov. & \% o Nac. & Dist. Prov. & \% Nac. \\
\hline \multirow{2}{*}{ Almería } & 28,3 & 3,1 & 22,4 & 0,5 & 59,2 & 0,7 \\
Cádiz & 11,3 & 3,0 & 29,8 & 1,8 & 58,8 & 2,0 \\
Córdoba & 15,0 & 2,6 & 27,6 & 1,0 & 57,3 & 1,3 \\
Granada & 11,8 & 2,0 & 22,4 & 0,8 & 65,7 & 1,4 \\
Huelva & 18,1 & 2,4 & 39,8 & 1,2 & 42,1 & 0,7 \\
Jaén & 17,8 & 2,5 & 29,9 & 0,9 & 52,2 & 0,9 \\
Málaga & 7,9 & 2,2 & 22,7 & 1,3 & 69,3 & 2,4 \\
Sevilla & 9,3 & 3,9 & 28,8 & 2,6 & 61,8 & 3,3 \\
ANDALUCIA & 12,9 & 21,7 & 27,7 & 10,1 & 59,3 & 12,8 \\
ESPAÑA & 7,4 & 100,0 & 34,4 & 100,0 & 58,1 & 100,0 \\
\hline
\end{tabular}

Fuente: Elaboración propia a partir de los datos de BANCO DE BILBAO, Renta Nacional, 1979.

Esta alta industrialización relativa del ámbito territorial que estamos considerando contrasta fuertemente con los niveles de desarrollo alcanzados no sólo en la escala nacional sino también en la regional. Aunque el concepto desarrollo es lo suficientemente amplio y ambigüo como para que resulte complejo delimitar con exactitud las variables que deben formar parte de su composición, existen indicadores macroeconómicos que, al menos en términos comparativos, ofrecen una aproximación cuantitativa de los niveles alcanzados. Es el caso por ejemplo, del índice nivel de desarrollo elaborado por el Anuario del Mercado Español (Banco Español de Crédito). Dicho índice, que engloba variables demográficas, culturales y propiamente económicas, ofrece para las provincias aldaluzas los valores expresados en el cuadro III. 
CUADRO III

NIVELES DE DESARROLLO (Med. Nac. $=100)$

\begin{tabular}{lllr}
\hline & & & \\
Almería & 88,3 & Huelva & 88,0 \\
Cádiz & 95,8 & Jaén & 83,9 \\
Córdoba & 94,2 & Málaga & 107,1 \\
Granada & 85,7 & Sevilla & 100,8 \\
\hline
\end{tabular}

Fuente: Anuario del Mercado Español, BANESTO, 1982.

Excepto Sevilla, que ocupa el segundo lugar de la región y que rebas mínimamente la media nacional, las otras dos provincias del Bajo Guadalqui vir no alcanzan un desarrollo acorde con el papel industrial que desempeñar en el contexto regional. En este sentido resalta con luz propia el caso di Huelva que constituye un claro exponente del desajuste entre los niveles d industrialización y desarrollo. La explicación hay que buscarla necesa riamente en la forma en que se concibió su Polo de Desarrollo. Surgido di una escueta filosofía de desarrollo a través de industrialización el Polo di Huelva ha carecido de la necesaria articulación con el resto de los sectore económicos de la región, al objeto de proyectar el crecimiento industria sobre su propio entorno natural ${ }^{1}{ }^{1}$. Esta filosofía de ubicación de islote industriales, sin una suficiente concertación o entrelazamiento con los sec tores y espacios circundantes, es extensiva a la mayor parte de la actividar productiva andaluza.

\section{CONSIDERACIONES SOBRE LA INTEGRACION ESPACIAL}

Además de la inexistencia de un tejido industrial congruentemente articu lado, se pueden señalar otros factores que obstaculizan en gran medida $\epsilon$ proceso de desarrollo que con frecuencia conlleva la dinámica de la industria lización. Uno de ellos es sin duda la ausencia de la infraestructura necesari que facilite las interconexiones entre los distintos centros comerciales y d producción, y que, por contra, propicia a medio y largo plazo la configur: ción de un desarrollo desequilibrado o polarizado.

11. NARBONA, C. y ROMAN, C. (1979): “Industrialización y desarrollo económico: el caso ( Huelva" Estudios Regionales, n. ${ }^{\circ} 4$, pp. 123-140. 
Desde esta perspectiva y dentro del marco provincial del Bajo Guadalquivir, la formación de los centros de influencia comercial parece localizarse en función del trazado de las más importantes líneas de comunicación existentes. Así, el Anuario del Mercado Español ${ }^{12}$ distingue siete cabeceras de áreas comerciales: Sevilla, Cádiz, Huelva, Jerez, Algeciras, La Línea y Ayamonte. Sus cuotas de mercado se reflejan en el cuadro IV.

\section{CUADRO IV}

CUOTAS DE MERCADO DE LAS AREAS COMERCIALES

DEL TRIANGULO SEVILLA, CADIZ Y HUELVA

(Total Nacional: 100.000)

\begin{tabular}{lrc}
\hline Provincias & Cuota & Orden Nacional $(*)$ \\
\hline Sevilla & 3.616 & 5 \\
Cádiz & 934 & 26 \\
Huelva & 661 & 36 \\
Jerez & 520 & 44 \\
Algeciras & 283 & 64 \\
La Línea & 130 & 87 \\
Ayamonte & 34 & 101 \\
\hline
\end{tabular}

(*) Sobre un total de 101 áreas comerciales españolas.

Fuente: Anuario del Mercado Español, BANESTO, 1981.

Estos centros gravitacionales de carácter comercial se alinean en su totalidad, en torno a las principales arterias que, casi en forma de V marcan un trazado con vértice en Sevilla. Prestando atención a la vía de comunicación más importante en el ámbito interno andaluz - la carretera alberga alrededor del $80 \%$ del tráfico comercial-, se pueden establecer algunas consideraciones de interés.

Obviamente por su hegemonía regional y por su apertura hacia el interior de la península, Sevilla se configura como auténtico centro de gravedad de las tres provincias. Desde esta capital parten hacia Cádiz las dos arterias más importantes en cuanto a categoría viaria, del sur de España: la autopista de peaje Sevilla-Cádiz - única existente en Andalucía- y la CN-IV -también la única con carácter radial para el sur de la peninsula-. De esta infraestructura comunicativa se benefician notablemente los núcleos industriales de la provincia de Cádiz: zona vinícola de Jerez-Sanlúcar, industrias del cuero en la Sierra y muy especialmente el litoral portuario de la bahía de Cádiz y Campo de Gibraltar. A la importancia adquirida ha contribuido sin duda,

12. Anuario del Mercado Español, Banesto 1981. En esta edición el estudio se hace para 101 áreas comerciales de todo el territorio español. 
además de su notorio enclave geoestratégico y el caracter fuertemente enraizado de las industrias tradicionales, la apertura por carretera hacia la Costa del Sol, a través de la CN-340.

Aunque de menor intensidad e inferior categoria viaria con respecto a Cádiz, la conexión Sevilla-Huelva cuenta en la actualidad con unas vias de comunicación de especial relevancia dentro de la red existente en Andalucía. Paralelamente a la antigua y deficiente $\mathrm{N}-341$ la nueva autovía A-49, ha supuesto un importante reforzamiento en la comunicación entre las dos capitales, con independencia, claro está, de que no se haya llegado a satisfacer plenamente la necesidad de una autopista que hace unos años estaba planteada. La prolongación de la via hacia Portugal cubre las necesidades comerciales derivadas de la pesca, así como las exigencias del turismo interior. Ello ha coadyuvado al auge de municipios como Punta Umbría, Lepe (La Antilla), Isla Cristina, y a la ya mencionada área comercial de Ayamonte, cuyo último lugar en el escalafón nacional (cuadro IV) no desdice de su importancia relativa con respecto a una amplia zona del oeste provincial.

Desde la óptica del dinamismo de los núcleos poblacionales, y según un estudio de reciente publicación ${ }^{13}$, -en el que se concibe el dinamismo en función del aumento de la población por encima de la media provincial-, además de las capitales, los municipios dinámicos de las tres provincias vienen a localizarse casi en su totalidad en el círculo de influencia económica de Sevilla y a lo largo de las principales arterias ya comentadas, que desde el Campo de Gibraltar y Ayamonte confluyen en el nodo sevillano. Este esquema de conexión, en lo que respecta a la provincia de Cádiz ha impulsado, o contribuido a impulsar, el dinamismo de municipios como Los Palacios, Las Cabezas de San Juan, Jerez, Rota, Puerto de Santa María, Puerto Real, San Fernando, Chiclana, Algeciras y San Roque. En tomo al otro lado de la $\mathrm{V}$ adquirieron dinamismo Ayamonte, Isla Cristina, Lepe, Punta Umbría, Palos, Moguer, San Juan del Puerto, Trigueros, Bollullos del Condado, Almonte, Sanlúcar la Mayor, Olivares, Bollullos Mitación y Villanueva del Ariscal, además de otras poblaciones lindantes al núcleo urbano de Sevilla $^{14}$. Fuera del cauce de influencias de estas dos principales vias de comunicación solo Ubrique en la provincia de Cádiz, y algunas ciudades próximas a Sevilla -en su mayoría como consecuencia del carácter residencial con respecto a ésta--, obtuvieron signos de dinamismo para el período 1960-75 que venimos considerando. En la provincia de Huelva ningún municipio no perteneciente a la franja rectilínea entre las dos capitales, adquire tal condición de dinámico.

13. PAYNO GAlVARRIATO, J.A. (Dir.) (1983) Claves para el desarrollo económico de Andalucía. Junta de Andalucía, Fundación Banco Exterior, Sevilla, pp. 275 y ss.

14. PAYNO, J.A. Ob. cit. Los aumentos de población se han calculado para los años 1960-75 y se consideran solo los municipios mayores de 5.000 habitantes. 
Por el contrario desde un punto de vista de regresividad ${ }^{15}$, casi todos los municipios recesivos de las tres provincias se encuentran ubicados en áreas de sierra o fuera de los accesos inmediatos a los dos principales trazados intracapitales. Unicamente en los casos de Cartaya, Gibraleón y Rociana en la provincia de Huelva, y Vejer, Tarifa y La Línea en Cádiz se puede decir que no existe un aprovechamiento de la infraestructura de comunicación con los grandes vértices económicos de la sub-región.

A esta situación de desequilibrio intrazonal, contribuye gravemente la ausencia de una articulación aceptable en las carreteras nacionales, asi como el deficiente estado de conservación y calidad de una gran parte de las vias comarcales y locales. En este sentido, quizás el caso de la provincia de Huelva es más que relevante al no contar durante mucho tiempo con una apertura medianamente eficiente hacia la denominada Ruta de la Plata, y sufrir un cuasi aislamiento con una sola comunicación de importancia hacia Sevilla.

Al objeto de soslayar esta realidad surgen en 1977 los proyectos de dos carreteras: la autovía Sevilla-Huelva A-49 - a que ya hemos hecho referencia-, y la que uniera Huelva con Cádiz por via costera. Especialmente la problemática se centró en esta segunda ruta y prueba de ello fue la abierta polémica que en ese mismo año apareció en la prensa durante varios meses.

La via costera se proyectó con $120 \mathrm{kms}$. de recorrido y una anchura de 7 ms. Su primera parte Huelva-Torre La Higuera (Matalascañas) con $48 \mathrm{kms}$. está en servicio, y en ella convergen los nodos del entorno de la capital onubense con los turísticos del litoral. Al mismo tiempo ha reforzado la conectividad de los poblados de El Abalario - Bodegones, Los Cabezudos, El Abalario, etc.-, ofreciéndoles una cómoda salida meridional hacia el área periurbana de Huelva. La segunda y tercera parte no se han efectuado. Se corresponden en la provincia de Huelva al tramo Matalascañas-Rio Guadalquivir con $27 \mathrm{kms}$., y tras rebasar el colector bético - una de las cuestiones básicas del proyecto--, completar su recorrido en la provincia gaditana enlazando Bonanza-Sanlúcar de Barrameda-Puerto de Sta. María-Cádiz con $45 \mathrm{kms}$.

La existencia de un área de repulsión humana en la desembocadura del Guadalquivir favoreció la conservación de geo y ecosistemas naturales en esta comarca. Pero la trayectoria repulsiva se truncó definitivamente a partir de 1960 , fecha en la que las marismas se colonizan demográfica y agrícolamente. Salvado el escollo marismeño las ocupaciones turísticas del litoral más próximo no se hicieron esperar.

El Proyecto de Promoción Turística de la Costa de Huelva (1965) vino a introducir la primera zonificación del espacio litoral y propició, al mismo

15. PAYNO, J.A. Ob. cit. Se consideran municipios recesivos los que pierden población en el período considerado, siendo mayores de 5.000 hab., y los que disminuyen su demografía a una tasa doble que la media, cuando sus correspondientes provincias tienen evoluciones negativas. 
tiempo, la creación de Centros de Interés Turísticos Nacionales (C.I.T.N.). E de Matalascañas está fechado en 1969, y en su primera fase se plantea par dar acogida a más de 30.000 habitantes en unas 700 Has. La potenciación d la segunda fase constructiva coincide con el proyecto de la carretera coster y con el robustecimiento del uso turístico del litoral: los proyectos d urbanización del médano de El Asperillo (900 Has. que acogerian a uno 54.000 personas) y del pinar de Las Marismillas (160 Has, con 1.600 hab: tantes) 16 .

\section{PLANTEAMIENTOS FINALES}

El Bajo Guadalquivir es un excelente modelo geográfico y socioeconóm: co para analizar la problemática de la articulación territorial ${ }^{17}$. En él se con citan por una parte una vertiente socioeconómica que exige para su desarrc llo una infraestructura comunicativa que articule el tejido de los sectores prc ductivos y de consumo, y fundamentalmente los centros poblacionales qu actúan como agentes en esa dinámica socioeconómica. Y junto a ello la exis tencia de espacios naturales a proteger, destacando por su tamaño Doñana El conflicto fundamental surgió a la sazón sobre la base de la carretera Hue] va-Cádiz y actualmente se prolonga en relación con las presiones urbanística en el médano de El Asperillo.

Como acabamos de decir no podemos por menos que pronunciarnos favor de toda articulación, pero no a costa del territorio.

De partida nos parece que las vias costeras nunca debieran trazarse e: una banda de $15-20 \mathrm{kms}$. paralelas al litoral, favoreciéndose por contra la penetraciones perpendiculares y dendritas secundarias. Con tal consideració se abordan además otras insuficiencias: la densimétrica y la de calida infraestructural. La potenciación de una red local y provincial evitaría 1 aparición de desiertos comarcales, y paliarían al mismo tiempo la perific: $d_{a d}{ }^{1}$ de nuestro sistema de comunicaciones. Tratando con espacios natur les, el reforzamiento del empleo de vias agropecuarias ${ }^{19}$-valorando capac dades de carga con impactos medioambientales-, es la base de la articulació territorial. De ahí que el modelo conectivo de El Abalario con ramale internos desde el vial Almonte-Matalascañas, y salida meridional, nos parezc muy interesante de ampliar y en su caso trasladarlo a situaciones afines. $\mathrm{N}$

16. Los datos brutos de oferta turística pueden verse en el Estudio de Planificación Turística de lo Municipios Costeros de Huelva (1978) Secretaría de Estado de Turismo, INITEC, S.A.

17. Este problema es igualmente tocado por J. OJEDA profesor del Departamento de Geografía, qu en la actualidad redacta su tesis doctoral.

18. PAYNO, J.A. Ob. cit.

19. MIGUEL RUIZ, J.A. y otros (1982): "Potenciación de los usos de las vias pecuarias" Estudio Territoriales, 5, pp. 39-68. 
así el proyecto de un eje Almonte-Roçina-Abalario-Torre del Loro, ya que el reforzar linealmente la conectividad abogaría por un robustecimiento de la ocupación urbanística del litoral - por lo demás junto a la base militar de Arenosillo-y prelitoral ${ }^{2} 0$.

Altamente beneficioso es asimismo potenciar las comunicaciones entre los focos difusores de economias externas afines, que en el Bajo Guadalquivir son los complejos vitivinícolas del Condado y Jerez-Sanlúcar de Barrameda, o las áreas regables del Guadalete y Guadalquivir.

Por último la conectividad supraregional, tanto más importante que la intra, debe ampliarse entedemos, sobre la base de dos pilares: 1) por la Ruta de la Plata entrelazando adecuada e internamente el cuadrilátero Ayamonte-Niebla-Valverde del Camino-Sanlucar del Guadiana, en el extremo occidental del Bajo Guadalquivir; 2) e incidiendo en la via marítima tanto por los ahorros de coste y tiempo, cuanto por la proyección geoeconómica del Estrecho.

Habida cuenta del complejo entramado que acabamos de esbozar, y vista la buena disposición de la Consejería de Infraestructura y Política Territorial de la Junta de Andalucía, apuntada en las convocatorias de jornadas de estudios sobre esta materia, entendemos que corresponde a dicho departamento el canalizar un nuevo enfoque al tratamiento de este latente problema.

20. E1 proyecto Almonte-Torre del Loro aparece explicitado en el Proyecto de PDTC de Doñana, citado anteriormente. 